\title{
Articulation of Surfaces for Bio-applications
}

\author{
Arvind Agarwal, Sudipta Seal, and Narendra B. Dahotre
}

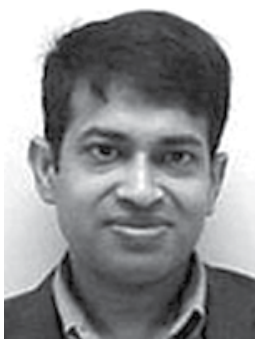

Arvind Agarwal

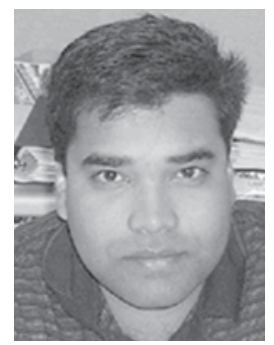

Sudipta Seal
Materials science and engineering is witnessing an increasing global focus on the materials related to energy, environment, and medical applications. The Surface Engineering Committee of the Materials Processing \& Manufacturing Division (MPMD) of TMS has been active in promoting the novel applications of emerging materials and technology. In 2008, the Surface Engineering Committee had a JOM theme of Materials and Surfaces for Energetics that dealt with subjects ranging from energetics of micro- and nano-scale materials to processing for energetic applications. This year, our committee has focused on Articulation of Surfaces for Bio Applications. The confluence of materials science with biology has opened up a plethora of opportunities for scientists and engineers to explore this exciting field of research. In this issue of JOM, the Surface Engineering Committee presents five papers that deal with different aspects of surface modifications for bio-applications.

The review by Ryan Boehm and coauthors provides an insight in the field of flexible electronics for biomedical applications. The authors have shown that polydimethylsiloxane (PDMS), diamond-like carbon (DLC), and gold materials which were used to make flexible electronics have adequate biocompatibility and may be used in medical applications such as neural probes,

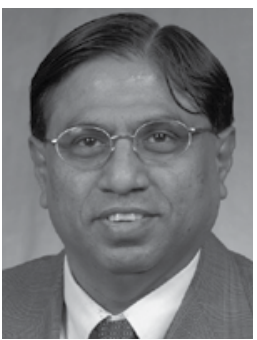

Narendra B. Dahotre arrays, and biosensors.

The paper by Kim and Pham focuses on understanding the interaction between human osteoblast cells and cathodic arc plasma deposited $\mathrm{CrN}$ thin film. Osteoblasts on $\mathrm{CrN}$ thin films had weakly distributed microtubules and no focal contact adhesion. The authors conclude that $\mathrm{CrN}$ thin film is a potential candidate as a protective coating on those implants that require minimal cellular adhesion.

The paper by Kantesh Balani et al. discusses the nano-scratch behavior of plasma sprayed hydroxyapatite (HA) coating reinforced with nano-aluminum oxide and carbon nanotubes (CNTs). The authors focus on understanding the effect of nanoparticle addition on the tribological properties of coating by observing a reduction in the wear volume by 13 times by nano-aluminum oxide addition. A total wear volume reduction of 65 times was observed with the combined addition of aluminum oxide and CNTs to HA coating. This improvement has been attributed to the reinforcement effect provided by $\mathrm{CNTs}$ and nano-aluminum oxide.

In another paper on hydroxypatite coatings, Liu et al. provide a review on a novel hybrid coating process that combines electrostatic spray technology with the microwave sintering process, for synthesizing HA-based nanostructured coating for dental and orthopedic implants. The authors synthesized HA coating with a controlled grain size from 50 to $300 \mathrm{~nm}$ with a gradient of nano- to micro-meter pore sizes to promote bone cell functions and to facili- tate bone healing. Preliminary in vitro study showed that the HA nanocoatings promoted human palatal mesenchymal cell functions and had improved microscratch resistance.

Strauss, Liu, and Camesano have presented their findings on bacterial adhesive behavior on protein surfaces. This study was performed using atomic force microscopy (AFM) imaging and quartz crystal microbalance with dissipation (QCM-D) techniques. An AFM probe coated with Staphylococcus epidermidis was used to probe a gold slide coated with FBS or fibronectin (FN) protein. The interaction with FBS was weak, but strong adhesion forces were observed between the bacteria and FN, which were attributed to biopolymers of the bacteria binding with the FN. The authors suggest that AFM and QCM-D are complementary methods to understand the complex interactions between bacteria, proteins, and coated surfaces.

The Surface Engineering Committee continues to promote the cause of novel materials, emerging technology and surface science/engineering through their symposium titled "Surface Engineering for Amorphous-, Nanocrystalline, and Bio-materials" in 2010 Annual TMS Meeting at Seattle, Washington.

Arvind Agarwal is an associate professor with the Department of Mechanical and Materials Engineering, Florida International University, Miami, Florida; Sudipta Seal is a professor in the Department of Mechanical, Materials, and Aerospace Engineering and director of the Advanced Materials Processing and Analysis Center, Nanoscience and Technology Center, Department of Mechanical, Materials, and Aerospace Engineering, at the University of Central Florida in Orlando, Florida; and Narendra B. Dahotre is a professor of Materials Science and Engineering in the Department of Materials Science and Engineering at the University of Tennessee, Knoxville, Tennessee. All are the advisors to JOM from the Surface Engineering Committee of the Materials Processing \& Manufacturing Division of TMS. 